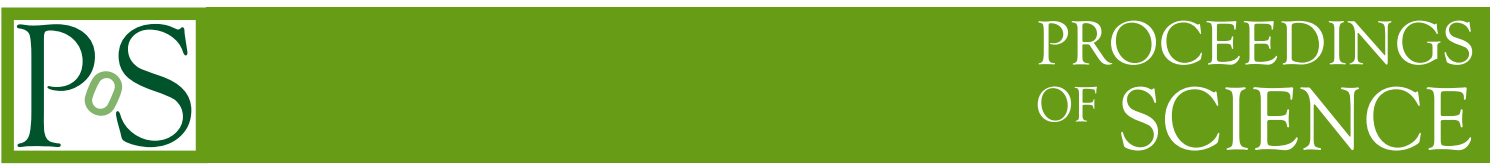

\title{
e-EVN monitoring of M87
}

\author{
M. Giroletti ${ }^{* a}$, G. Giovannini ${ }^{a, b}$, C. Casadio ${ }^{a}$, M. Beilicke $^{c}$, A. Cesarini ${ }^{d}$, and H. \\ Krawczynski ${ }^{c}$ \\ ${ }^{a}$ INAF Istituto di Radioastronomia, via Gobetti 101, 40129 Bologna (Italy) \\ E-mail: giroletti@ira.inaf.it \\ ${ }^{b}$ Dipartimento di Astronomia, via Ranzani 1, 40127 Bologna, Italy \\ ${ }^{c}$ Department of Physics, Washington University, St. Louis, MO 63130, USA \\ ${ }^{d}$ School of Physics, National University of Ireland, University Road, Galway, Republic of Ireland
}

\begin{abstract}
M87 is a privileged laboratory for a detailed study of the properties of jets, owing to its proximity $(\mathrm{D}=16.7 \mathrm{Mpc}, 1 \mathrm{mas}=0.080 \mathrm{pc})$, its massive black hole $\left(\sim 6.0 \times 10^{9} M_{\odot}\right)$ and its conspicuous emission at radio wavelengths and above. We started on November 2009 a monitoring program with the e-EVN at $5 \mathrm{GHz}$, in correspondence of the season of Very High Energy (VHE) observations. Indeed, two episodes of VHE activity have been reported in February and April 2010. We present here the main results of these multi-epoch observations: the inner jet and HST-1 are both detected and resolved in our datasets. We study the apparent velocity of HST-1, which seems to be increasing since 2005, and the flux density variability in the inner jet. All in all, the radio counterpart to this year's VHE event seems to be different from the ones in 2005 and 2008, opening new scenario for the radio-high energy connection.
\end{abstract}

10th European VLBI Network Symposium and EVN Users Meeting: VLBI and the new generation of radio arrays

September 20-24, 2010

Manchester Uk

\footnotetext{
* Speaker.
} 


\section{Introduction}

The galaxy Messier 87 (M87, or Virgo A) is the host to one of the nearest radio loud Active Galactic Nuclei (AGNs). It is located at the center of the Virgo cluster of galaxies at a distance $d=16.7 \mathrm{Mpc}$, resulting in an angular scale of 1 mas $=0.081 \mathrm{pc}$. The estimated mass of the huge black hole at its center is $M_{\mathrm{BH}}=6.4 \times 10^{9} M_{\odot}$ [9]. Combined with the low distance of the source, this implies a physical scale of 1 mas $=140 R_{\text {Schw }}$.

At radio wavelengths, M87 is associated with the bright source $3 \mathrm{C} 274$, characterized by a monochromatic luminosity of $\sim 10^{25} \mathrm{~W} \mathrm{~Hz}^{-1}$ (at $408 \mathrm{MHz}$ ). Its morphology is rather complex, with an extended halo visible on large angular scale [18] and an inner double lobe structure with a prominent one-sided jet. The jet extends for a few kiloparsecs and it is characterized by many substructures and knots. It has been well studied with the VLA, revealing superluminal proper motion of components [6]. At the high resolution offered by VLBI, the inner portion of the jet shows a limb brightened structure [13, 12, 14], while proper motion of components is only detected within the so-called HST-1 complex about 70 pc (projected) downstream [8].

At higher energy, the jet is also resolved by optical (HST) and X-rays (Chandra) observations, while gamma-ray telescopes reveal emission consistent with the position of M87 but do not resolve it, neither in the $\mathrm{MeV} / \mathrm{GeV}$ band from space with Fermi [1], nor in the $\mathrm{GeV} / \mathrm{TeV}$ one with ground based Cherenkov telescopes $[4,3,5]$.

Various models have been proposed to explain the multi-wavelength emission and in particular to constrain the site of the VHE emission in M87. The inner jet region seems favoured by the observed short TeV variability timescales [4]. The VHE emission could then be produced in the BH magnetosphere [16] or in the slower jet layer [19], with the spine accounting for the emission from the radio to the $\mathrm{GeV}$ band; this would lead to a complex correlation between the $\mathrm{TeV}$ and radio components. Later on, the episode of VHE activity observed in 2005 were accompanied by a dramatic increase of flux density in HST-1 at radio, optical, and X-ray energy. Given the compactness and superluminal motions observed in HST-1 itself with the VLBA, it was suggested that the TeV emission from M87 was originated in HST-1 [8, 11]. Finally, a strong TeV flare was detected again from M87 in February 2008, at the same time as a longer time scale increase of the radio flux from the nucleus, thus reintroducing the scenario in which the $\mathrm{TeV}$ flares originate in the core region [2].

The need for an additional high resolution coordinated multi-wavelength campaign targeting M87 was then pressing. In this paper, we present the contribution of the EVN to the efforts aimed at solving this puzzle of great astrophysical interest.

\section{Observations and Data Reduction}

In the above described context, we started a project to observe M87 with VLBI so that we could simultaneously reveal the properties of the M87 core, jet, and HST-1 structure. In order to grant the best possible combination of high sensitivity, good resolution, and large field of view, we used the EVN at $5 \mathrm{GHz}$. The observations have been carried out in e-VLBI mode, which delivers prompt results and offers a better time sampling than the regular EVN disk based sessions. 


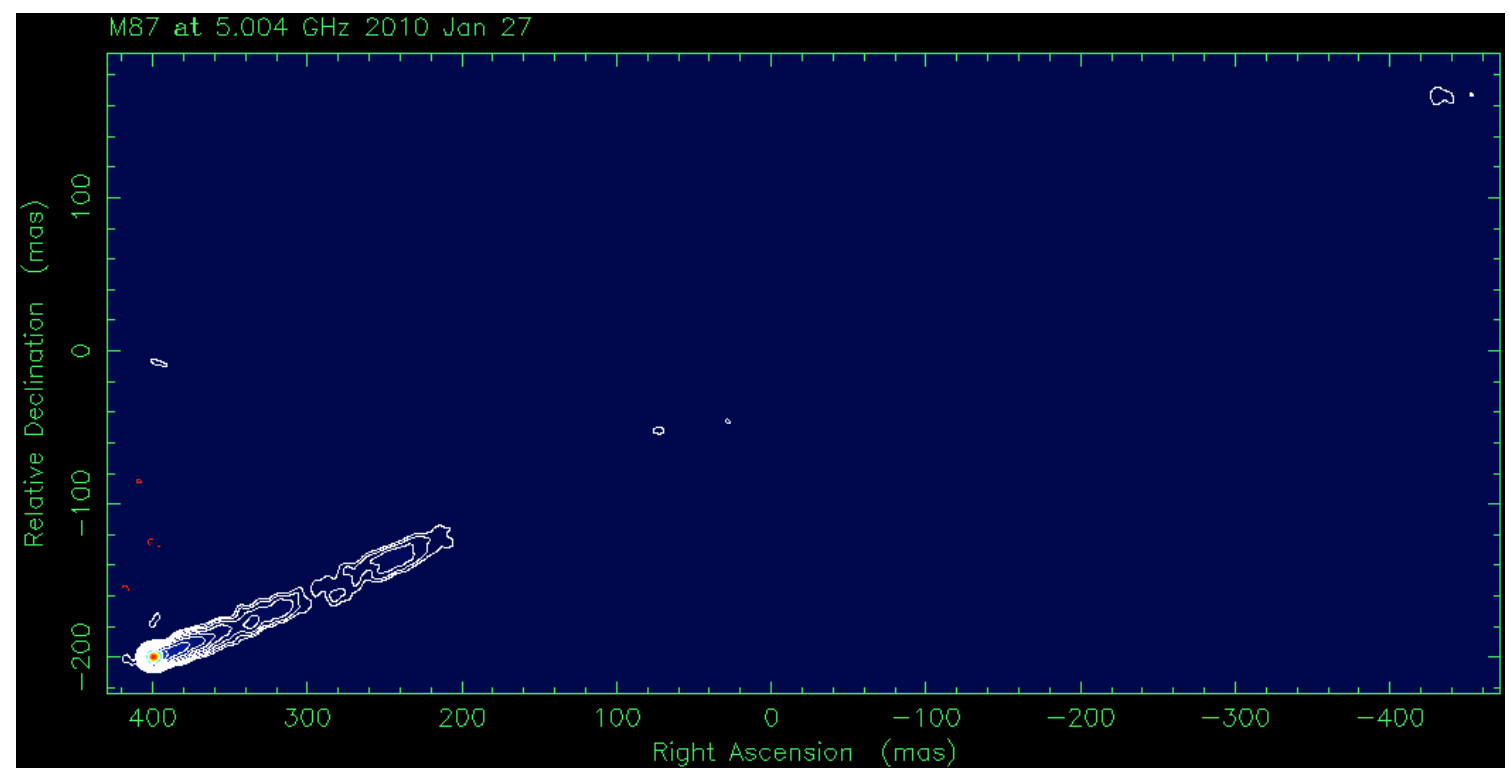

Figure 1: Wide field EVN image of the jet in M87. Contours are traced at $(-1,1,2,4, \ldots) \times 2.0 \mathrm{mJy} \mathrm{beam}^{-1}$. The peak flux density is $2 \mathrm{Jy}_{\text {beam }}^{-1}$, the restoring beam is $7.1 \times 6.6$ mas in PA $43^{\circ}$.

Observations were initially scheduled for 4 epochs, and later extended for a total of 7 observations between 2009 November and 2010 June. A further continuation of the project is also underway. The participating stations are Arecibo, Cambridge, Effelsberg, Jodrell Bank, Medicina, Onsala, Shanghai, Torun, and Westerbork. Data acquired from these telescopes were directly streamed to the central data processor at JIVE, and correlated in real-time. Pipeline calibration was carried out at JIVE and data were downloaded for additional editing and self calibration in AIPS and Difmap.

For observations taking advantage of the long baselines provided by the Arecibo and Shanghai telescopes, our clean beam with uniform weights is about $2.0 \times 0.9$ mas in PA $-25^{\circ}$. As a result of the large bandwidth (a rate of $1 \mathrm{Gbps}$ is sustained by most stations), long exposure (up to 6 hours per epoch), and extended collecting area, the rms noise in our images is mostly dynamic range limited. As an average value, we can quote $0.5-0.8 \mathrm{mJy} \mathrm{beam}^{-1}$ in the nuclear region and $0.1-$ $0.2 \mathrm{mJy}$ beam $^{-1}$ in the HST-1 region.

\section{Results}

Both the inner jet and the HST-1 region were well detected and imaged with our observational setup. A wide field $(900 \times 450$ mas) is shown in Fig. 1, while a zoom on HST-1 is presented in Fig. 2. The well known structure of the jet is confirmed, with emission detected for over 300 mas from the core in position angle PA $\sim-65^{\circ}$. HST-1 is revealed at $\sim 920$ mas from the core, aligned with the inner jet but oriented in PA $\sim-90^{\circ}$. HST-1 itself is resolved into several components, the brightest and most compact of which is located upstream the rest of emission.

The observations were scheduled so that they would take place during the season of best conditions for the $\mathrm{TeV}$ observatories. Indeed, two episodes of VHE enhanced activity have been reported during our campaign, one on 2010 February 9th [15] and one starting on April 8th [17]. 


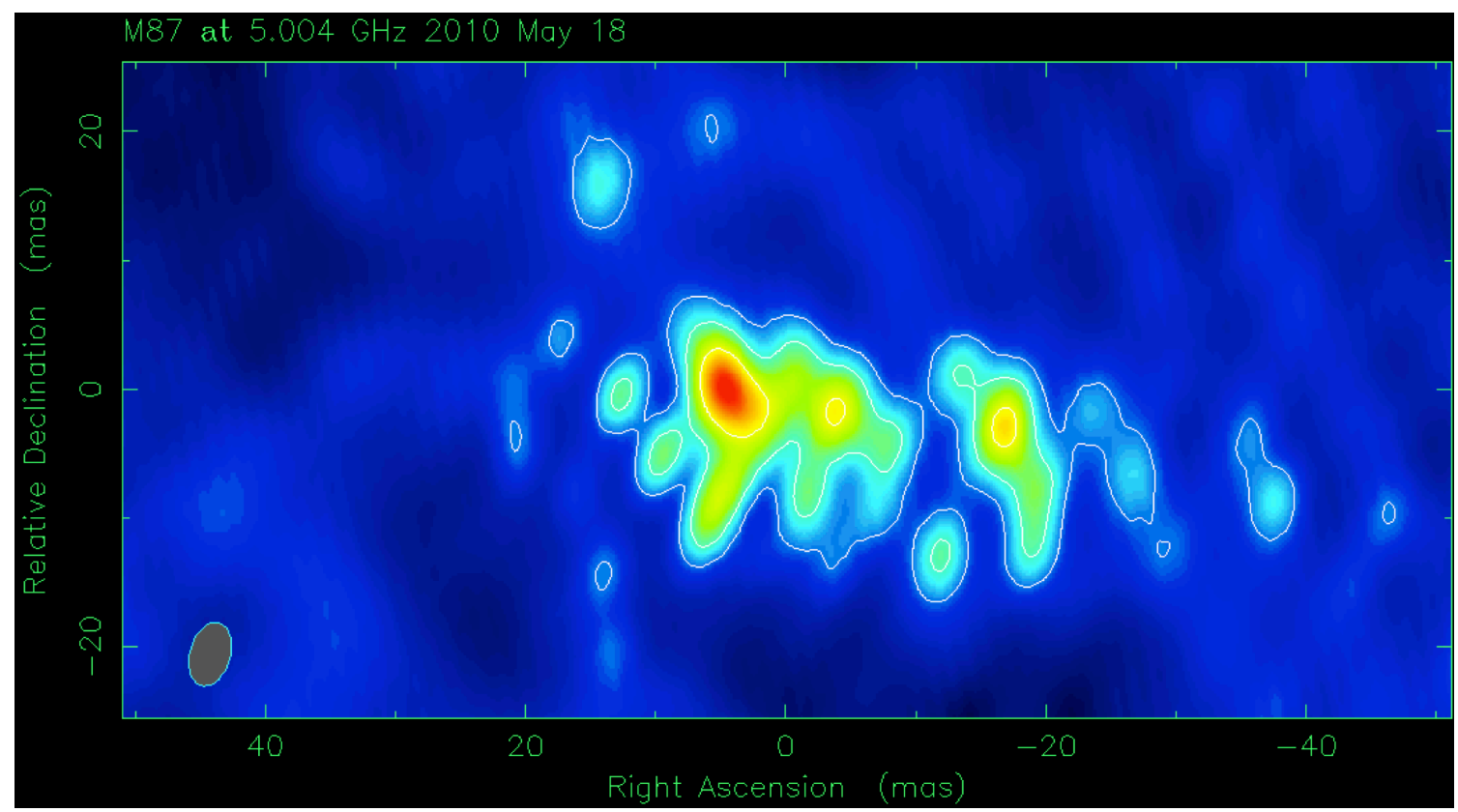

Figure 2: EVN image of the HST-1 region downstream the jet in M87 on 2010 May 18. Contours are traced at $(-0.3,0.3,0.6,1.2) \mathrm{mJy}_{\text {beam }}{ }^{-1}$. The peak flux density is $2.0 \mathrm{mJy}$ beam $^{-1}$, the restoring beam is $5.0 \times 3.1$ mas in $\mathrm{PA}-13^{\circ}$.

\begin{tabular}{lll}
\hline Epoch & $\begin{array}{l}\text { Core flux } \\
(\mathrm{Jy})\end{array}$ & $\begin{array}{l}\text { HST-1 Peak } \\
\left(\mathrm{mJy} \mathrm{beam}^{-1}\right)\end{array}$ \\
\hline 2009 Nov 19 & $1.81 \pm 0.03$ & $3.5 \pm 0.2$ \\
2010 Jan 27 & $1.81 \pm 0.03$ & $2.7 \pm 0.2$ \\
2010 Feb 10 $\left(^{*}\right)$ & $1.80 \pm 0.03$ & $3.0 \pm 0.2$ \\
2010 Mar 6 & $1.89 \pm 0.03$ & $4.6 \pm 0.3$ \\
2010 Mar 28 $(*)$ & $2.01 \pm 0.03$ & $3.4 \pm 0.2$ \\
2010 May 18 & $1.93 \pm 0.03$ & $2.8 \pm 0.2$ \\
2010 Jun 9 & $1.93 \pm 0.03$ & $2.6 \pm 0.2$ \\
\hline
\end{tabular}

Table 1: Preliminary core flux density and HST-1 peak brightness at $5 \mathrm{GHz}$ during the EVN monitoring campaign. Epochs marked by an asterisk are those next to reported events of VHE activity (Feb 9th and Apr 8-10 [15, 17]).

The first flare is almost simultaneous (within 24hr) to our third EVN epoch. Our image from that epoch shows that the core and HST-1 structure are still comparable to the pre-flare values and provide a good reference for light curve and proper motion studies of jet substructures with respect to the time of the VHE event [10]. As a matter of fact, even in later epochs both the core and HST-1 have remained on similar values of flux density. A quick summary is reported in Table 1. Even if the values are still somewhat preliminary, it is clear that no dramatic variability is present.

Although the structure of HST-1 is clearly rather complex and overall quite weak, it is still possible to identify and follow epoch by epoch at least the brightest and most compact component. Fixing the core as a reference, we find that the peak is moving with an apparent velocity of $v_{\text {app }} \sim 3 c$ 


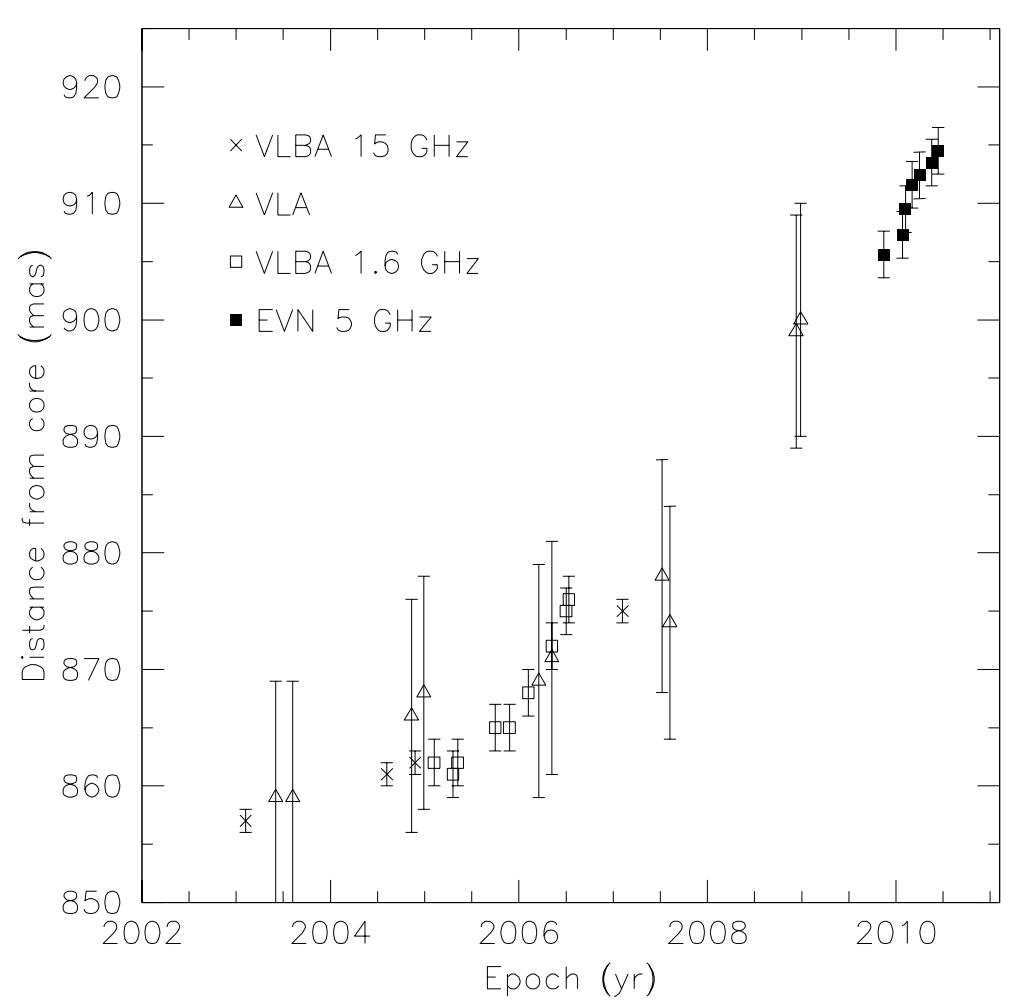

Figure 3: Distance of HST-1 peak from the core in M87, from new EVN data (filled squares) combined with literature VLBA data at 1.6 [8] and $15 \mathrm{GHz}$ [7] and from the VLA archive.

and it is thus superluminal. No stationary component seems to be present in our images.

To put this result in context, we have obtained from the literature $[8,7]$ and the archives some additional images in which HST-1 is detected. The images have different resolutions and frequency, so a comparison is not trivial. However, it is clear that there is an overall trend of increase in separation between HST-1 and the core, as shown in Fig. 3. The data suggest a change in the proper motion velocity at the epoch $\sim 2005.5$, coincident with the $\mathrm{TeV}$ activity and the peak in the VLA, HST, and Chandra light curves of HST-1. In particular, the apparent velocity is $\sim 0.5 c$ in the time range $2003-2005.5$, while it becomes $\sim 2.7 c$ on the period $2005.5-2010.25$. Further variations could be present, such as a decrease in the apparent velocity in 2007 with a restarted high velocity motion from 2008 (near the time of the high energy flare) up to now. Assuming a jet orientation angle $=25^{\circ}$ a proper motion of $2.7 c$ corresponds to an intrinsic velocity $=0.94 \mathrm{c}$.

\section{Discussion and Outlook}

Assuming the VHE events in February and April 2010 are representative of the same state of high activity, we have three different episodes to analyse and compare, in 2005, 2008, and 2010. At lower energy, a simultaenous increase of flux density was found in one case in HST-1 (for 2005), in one case in the core (2008), and in neither HST-1 nor the core for the latest event (2010). Indeed, even if it is natural to expect a delay in the $5 \mathrm{GHz}$ data, the images taken as late as June (more 
than 4 months after the first VHE event) do not reveal any significant enhancement of the radio flux density.

The situation is thus certainly complex; one possibility is that the three events have actually different physical origins. It is however a little surprising that the conditions for such extremely energetic events can be reached from rather different starting conditions. On the other hand, there is the possibility that none of the low energy increase is actually related to the $\mathrm{TeV}$ events, which would then be totally unrelated to the radio light curve.

Finally, it is possible that the interplay between the VHE and radio emission is taking place in a more complex way. In this sense, it might be worth considering the hint given by the proper motion study, which suggests that the VHE events could be related to an increase in the apparent velocity of HST-1. Although the current data are still inadequate to support such claim, it could probably be confirmed or disproved with a longer monitoring.

In order to clarify the overall picture, it is thus important to continue to monitor the source from radio to $\mathrm{TeV}$. Moreover, a detailed look at the archives can provide an improved understanding of the behavior of the source in the past.

\section{Acknowledgments}

MG acknowledges financial contribution from ASI-INAF I/088/06/0. The European VLBI Network is a joint facility of European, Chinese, South African and other radio astronomy institutes funded by their national research councils.

\section{References}

[1] Abdo, A. A., Ackermann, M., Ajello, M., et al. Fermi Large Area Telescope Gamma-Ray Detection of the Radio Galaxy M87 2009, ApJ, 707, 55, [arXiv:0910.3565]

[2] Acciari, V. A., Aliu, E., Arlen, T., et al. Radio Imaging of the Very-High-Energy $\gamma$-Ray Emission Region in the Central Engine of a Radio Galaxy 2009, Sci, 325, 444, [arXiv:0908.0511]

[3] Acciari, V. A., Beilicke, M., Blaylock, G., et al. Observation of Gamma-Ray Emission from the Galaxy M87 above $250 \mathrm{GeV}$ with VERITAS 2008, ApJ, 679, 397, [arXiv:0802.1951]

[4] Aharonian, F., Akhperjanian, A. G., Bazer-Bachi, A. R., et al. Fast Variability of Tera-Electron Volt $\gamma$ Rays from the Radio Galaxy M87 2006, Sci, 314, 1424, [arXiv:astro-ph/0612016]

[5] Albert, J., Aliu, E., Anderhub, H., et al. Very High Energy Gamma-Ray Observations of Strong Flaring Activity in M87 in 2008 February 2008, ApJ, 685, L23, [arXiv:0806.0988]

[6] Biretta, J. A., Zhou, F., Owen, F. N. Detection of Proper Motions in the M87 Jet 1995, ApJ, 447, 582

[7] Chang, C. S., Ros, E., Kovalev, Y. Y., Lister, M. L. VLBI detection of the HST-1 feature in the M 87 jet at $2 \mathrm{~cm}$ 2010, A\&A, 515, A38, [arXiv:1002.2588]

[8] Cheung, C. C., Harris, D. E., Stawarz, Ł. Superluminal Radio Features in the M87 Jet and the Site of Flaring TeV Gamma-Ray Emission 2007, ApJ, 663, L65, [arXiv:0705.2448]

[9] Gebhardt, K., Thomas, J. The Black Hole Mass, Stellar Mass-to-Light Ratio, and Dark Halo in M87 2009, ApJ, 700, 1690, [arXiv:0906.1492] 
[10] Giroletti, M., Giovannini, G., Beilicke, M., Cesarini, A., Krawczynski, H. EVN observations of the radio galaxy M87 following a TeV flare 2010, ATel, 2437, 1

[11] Harris, D. E., Cheung, C. C., Stawarz, Ł., Biretta, J. A., Perlman, E. S. Variability Timescales in the M87 Jet: Signatures of $E^{2}$ Losses, Discovery of a Quasi Period in HST-1, and the Site of TeV Flaring 2009, ApJ, 699, 305, [arXiv:0904.3925]

[12] Kovalev, Y. Y., Lister, M. L., Homan, D. C., Kellermann, K. I. The Inner Jet of the Radio Galaxy M87 2007, ApJ, 668, L27, [arXiv:0708.2695]

[13] Krichbaum, T. P., Agudo, I., Bach, U., Witzel, A., Zensus, J. A. VLBI at the highest frequencies - AGN studies with micro-arcsecond resolution 2006, POS (8thEVN) 002

[14] Ly, C., Walker, R. C., Junor, W. High-Frequency VLBI Imaging of the Jet Base of M87 2007, ApJ, 660, 200, [arXiv:astro-ph/0701511]

[15] Mariotti, M. MAGIC observes increased VHE gamma ray flux from M87 2010, ATel, 2431, 1

[16] Neronov, A., Aharonian, F. A. Production of TeV Gamma Radiation in the Vicinity of the Supermassive Black Hole in the Giant Radio Galaxy M87 2007, ApJ, 671, 85, [arXiv:0704.3282]

[17] Ong, R. A., Mariotti, M. VERITAS and MAGIC Report Flaring in Very High Energy Gamma Rays from M87 2010, ATel, 2542, 1

[18] Owen, F. N., Eilek, J. A., Kassim, N. E. M87 at 90 Centimeters: A Different Picture 2000, ApJ, 543, 611, [arXiv:astro-ph/0006150]

[19] Tavecchio, F., Ghisellini, G. Spine-sheath layer radiative interplay in subparsec-scale jets and the TeV emission from M87 2008, MNRAS, 385, L98, [arXiv:0801.0593] 